\title{
Effectiveness of Influenza Vaccine for Preventing Laboratory-Confirmed Influenza Hospitalizations in Immunocompromised Adults
}

Kailey Hughes ${ }^{1}$, Donald B Middleton ${ }^{1,2}$, Mary Patricia Nowalk², Goundappa K Balasubramani², Emily T Martin ${ }^{3}$, Manjusha Gaglani ${ }^{4}, \mathrm{H}$ Keipp Talbot ${ }^{5}$, Manish M Patel ${ }^{6}$, Jill M Ferdinands ${ }^{6}$, Richard K Zimmerman ${ }^{1,2}$, Fernanda P Silveira ${ }^{1,2}$, for the HAIVEN Study Investigators

1 University of Pittsburgh Medical Center, Pittsburgh, PA

2 University of Pittsburgh, Pittsburgh, PA

3 University of Michigan School of Public Health, Ann Arbor, MI

4 Baylor Scott \& White Health, Texas A\&M University College of Medicine, Temple, TX

5 Vanderbilt University Medical Center, Nashville, TN

6 Influenza Division, Centers for Disease Control and Prevention, Atlanta, GA

\section{Corresponding author:}

Fernanda P. Silveira, MD, MS, FIDSA

Associate Professor of Medicine

3601 Fifth Avenue, Suite 5B

Pittsburgh, PA 15213

Phone: 412-648-6601

Fax: 412-648-6399

Email: silveirafd@upmc.edu

\section{Alternate corresponding author:}

Kailey Hughes, MPH

3601 Fifth Avenue, Suite 5B

Pittsburgh, PA 15213

Phone: 412-648-6453

Fax: 412-648-6399

Email: hugheskl4@upmc.edu

Word count: Text 2982; Abstract 247; Tables 3; Figures 2

Keywords: influenza; vaccine; vaccine effectiveness; immunocompromised

Running title: Flu VE in immunocompromised adults 


\section{ABSTRACT}

Background: Yearly influenza immunization is recommended for immunocompromised (IC) individuals, although immune responses are lower than that for the non-immunocompromised and the data on vaccine effectiveness (VE) in the IC is scarce. We evaluated VE against influenza-associated hospitalization among IC adults.

Methods: We analyzed data from adults $\geq 18$ years hospitalized with acute respiratory illness (ARI) during the 2017-2018 influenza season at 10 hospitals in the United States. IC adults were identified using pre-specified case-definitions, utilizing electronic medical record data. VE was evaluated with a test-negative case-control design using multivariate logistic regression with PCR-confirmed influenza as the outcome and vaccination status as the exposure, adjusting for age, enrolling site, illness onset date, race, days from onset to specimen collection, selfreported health, and self-reported hospitalizations.

Results: Of 3,524 adults hospitalized with ARI, 1,210 (34.3\%) had an immunocompromising condition. IC adults were more likely to be vaccinated than non-IC (69.5\% vs $65.2 \%)$, and less likely to have influenza (22\% vs $27.8 \%)$. The mean age did not differ among IC and non-IC (61.4 vs 60.8 years old). The overall VE against influenza hospitalization, including immunocompetent adults, was $33 \%(95 \% \mathrm{Cl}, 21 \%$ to $44 \%)$. VE among IC vs non-IC adults was lower at $5 \%(-29 \%$ to $31 \%)$ vs. $41 \%(27 \%$ to $52 \%)(p<0.05$ for interaction term).

Conclusions: VE in one influenza season was very low among IC individuals. Future efforts should include evaluation of VE among the different immunocompromising conditions and whether enhanced vaccines improve the suboptimal effectiveness among the immunocompromised. 
medRxiv preprint doi: https://doi.org/10.1101/2020.10.08.20208579; this version posted October 13, 2020. The copyright holder for this preprint (which was not certified by peer review) is the author/funder, who has granted medRxiv a license to display the preprint in perpetuity.

It is made available under a CC-BY-NC-ND 4.0 International license .

\section{Introduction}

The number of immunocompromised (IC) individuals has increased due to greater longevity of the population, increasing numbers of solid organ and stem cell transplants, advances in the treatment of hematologic and solid malignancies, increase in the number of individuals living with human immunodeficiency virus (HIV), and the use of steroids, immune-modulating agents, and other immunosuppressive drugs to treat autoimmune and inflammatory conditions [1, 2]. Immunosuppressive conditions are heterogeneous and the degree and type of immune deficiency caused by each one of these conditions vary, but a unifying consequence is an increased risk of many infectious diseases including influenza [3]. Influenza is a common cause of illness and death, with an estimated 140,000-810,000 influenza-associated hospitalizations and 12,000-61,000 influenza-associated deaths annually in the United States [4].

IC individuals are at higher risk for influenza-related complications, including increased frequency of hospitalization, ICU admission, longer duration of hospitalization, and death [5-10]. Influenza vaccination is the best available intervention for preventing these complications and annual influenza vaccination is recommended for IC individuals [11]. However, the data on protection afforded by influenza vaccines in IC adults are scarce. A recent study on cancer patients demonstrated a vaccine effectiveness (VE) of $20 \%$ against influenza hospitalization, as compared to $42 \%$ in the general population $[12,13]$. Most studies of IC adults are small and evaluate immunogenicity as a surrogate of effectiveness [14]. These immunogenicity studies among various IC groups have demonstrated that antibody responses to inactivated influenza vaccines are suboptimal compared to those without immunosuppression $[14,15]$. However, immune response to vaccine does not necessarily directly relate to vaccine effectiveness [16, 17]. Since the 2015-2016 influenza season, the Centers for Disease Control and Prevention (CDC)-funded U.S. Hospitalized Adult Influenza Vaccine Effectiveness Network (HAIVEN) has estimated influenza VE among adults hospitalized for acute respiratory infections. 
Understanding influenza VE in IC individuals is crucial to the development of appropriate vaccination and public health policies. The purpose of this study was to evaluate influenza VE among hospitalized immunocompromised adults enrolled in the HAIVEN study during the 20172018 influenza season, when specific efforts were made to identify immunocompromised patients using case-definitions for immunocompromising conditions.

\section{Methods}

\section{Study design and enrollment}

The HAIVEN study is a multi-center, prospective, test-negative case-control study to determine an annual estimate of VE against influenza-associated hospitalizations among adults in the United States. Methods for the HAIVEN study have been described previously[18]. Briefly, adults $\geq 18$ years of age with new or worsening cough or sputum production of $\leq 10$ days' duration and a respiratory specimen collected $\leq 10$ days from illness onset and $\leq 72$ hours after hospital admission at one of ten hospitals in Pennsylvania, Michigan, Tennessee, and Texas were eligible. Inclusion criteria included age $\geq 18$ years, admission for an acute respiratory illness, or worsening of a chronic respiratory illness with a new or worsening cough. During the 2017-2018 influenza season, details on demographics, symptoms, influenza vaccination status, number of recent hospitalizations, and history of organ or stem cell transplant and, chemotherapy or radiation therapy in the preceding year were collected through the enrollment interview. Information about the clinical course and disease severity was obtained from electronic medical records (EMR). All international classification of diseases-10 diagnosis clinical modification (ICD-10-CM) codes and current procedural terminology (CPT) codes from all encounters in the 12 months before enrollment were obtained from the EMR and utilized to identify the high-risk conditions associated with an increased risk of serious influenza complications [11]. 
medRxiv preprint doi: https://doi.org/10.1101/2020.10.08.20208579; this version posted October 13, 2020. The copyright holder for this preprint (which was not certified by peer review) is the author/funder, who has granted medRxiv a license to display the preprint in perpetuity.

It is made available under a CC-BY-NC-ND 4.0 International license .

\section{Influenza case classification}

Enrolled patients provided respiratory specimens for influenza testing by polymerase chain reaction (PCR). Specimens were either nasal and oropharyngeal swabs that were tested in research laboratories with CDC PCR protocols or clinical nasopharyngeal specimens tested by PCR in hospital laboratories provided they were collected within 10 days of illness onset and 72 hours of admission. Enrolled patients who tested positive for influenza were classified as cases and those who tested negative for all influenza types were controls.

\section{Influenza vaccination status}

Self-reported current season influenza vaccination status was confirmed by medical record review, state immunization registry records, occupational health records, health insurance billing claims, and records from patients' primary care providers. Information collected included date and route of administration and product name, manufacturer, and lot number. Self-reported vaccination was accepted if the patient provided a date and location for the vaccination. A participant was considered vaccinated if s/he received the 2017-18 influenza vaccine $\geq 14$ days before illness onset. Because up to 14 days is required to mount an immune response to vaccination, those vaccinated 0 to 13 days before illness onset were excluded due to indeterminate vaccination status.

\section{Identification of immunocompromising conditions}

All ICD-10-CM codes for all encounters and receipt of the biologic chemotherapeutic agents bortezomib, carfilzomib, daratumumab, dasatinib, gemtuzumab, and imatinib in the year before study enrollment were collected from EMR data. In the 2017-2018 influenza season the enrollment questionnaire asked if the participant received chemotherapy or radiation therapy for cancer in the 12 months before enrollment. Eight groups of immunocompromising conditions were defined: organ transplantation, stem cell transplantation, underlying immunodeficiency, 
connective tissue disorder, receipt of chemotherapy or radiation therapy, hematologic conditions, chronic steroid use, and HIV. The basis for the groups was a previously described algorithm for identifying patients with active immunosuppression utilizing ICD and CPT codes in a large database of patients with severe sepsis[19]. We slightly modified this algorithm in two aspects. For solid malignancies, we only included patients actively treated with chemotherapy or radiation to improve specificity of immunosuppression. We also included patients with chronic use of steroids. We considered the enrollment question on receipt of chemotherapy or radiation therapy as the gold standard and our data found that ICD-10-CM and CPT codes have low sensitivity to identify patients receiving chemotherapy or radiation therapy (Supplementary Table S1). Therefore, we identified patients with immunocompromising conditions based on ICD-10-CM codes listed (Table 1 and Supplementary Table S2), except for the receipt of chemotherapy or radiation therapy, which were determined from ICD-10-CM codes, or receipt of one of the biologic chemotherapeutic agents listed, or a positive answer to the enrollment question about the receipt of chemotherapy or radiation therapy.

The IC groups were mutually exclusive; therefore, if a participant had more than one IC condition, they were grouped hierarchically following the group order listed above. The hierarchical order of groups is shown in supplementary Figure S1 and was based on the authors' expert opinion, to better identify active immunosuppressive conditions because we did not have data on the use of immunosuppressants other than steroids and biologicals in the dataset. For example, to identify patients with malignancies on active therapy, chemotherapy or radiation therapy preceded hematologic condition.

\section{Statistical analysis}

Demographic and other characteristics of the IC and non-IC groups were compared using Pearson $\chi^{2}$ test or Fisher exact test for categorical variables and two-sample t-test for continuous variables. 
VE was calculated by estimating the odds of influenza positivity among vaccinated patients compared to unvaccinated patients for the IC and non-IC groups using multivariate logistic regression using influenza positivity as the outcome and vaccination status as the exposure variable, with VE $=(1-$ adjusted odds ratio $) \times 100 \%[20]$

In the primary analysis, we stratified the sample by immunocompromised status and estimated VE in each stratum:

For $i=1$ to 2 strata of overall (any) immunocompromising status,

$$
\operatorname{logit}(f l u=1)=\beta_{0}+\beta_{1}(\operatorname{vacc}) \ldots+\beta_{Z} Z
$$

where

flu $=1$ if PCR-confirmed flu case (of specific type/subtype); 0 otherwise

vacc $=1$ if received vaccine $\geq 14 \mathrm{~d}$ prior to symptom onset; 0 otherwise

$Z=\quad$ = vector of adjustment variables including age (continuous), enrollment site, race, days from illness onset to specimen collection, date of illness onset (categorized as pre-peak, peak, or post-peak influenza periods [18]), self-reported health status (poor/fair and good/very good/excellent) and self-reported number of hospitalizations

and with VE defined as

$$
\widehat{V E}=\left[1-\exp \left(\beta_{1}\right)\right] * 100 \% \text {. }
$$

To test if VE differed by immunocompromised status, we regressed flu status on vaccination status, immunocompromised status, and the pairwise multiplicative interaction between vaccination status and immunocompromised status:

$$
\operatorname{logit}(\text { flu }=1)=\beta_{0}+\beta_{1}(\text { vacc })+\beta_{2}(I C)+\beta_{3}(\text { vacc } * I C) \ldots+\beta_{Z} Z
$$


where variables are defined as above and

IC = $\quad 1$ if immunocompromised (any immunocompromising condition); 0 otherwise

Effect modification of VE by immunocompromised status was assumed to be statistically significant if the test statistic for assessing if the coefficient for the interaction term, $\beta_{3}$, differed from zero had a $p$ value $<0.05$.

In secondary analyses, we stratified subjects by type-specific immunocompromised status and estimated VE within each stratum using a main effects model:

For $i=1$ to 9 strata of type - specific immunocompromising status,

$$
\operatorname{logit}(f l u=1)=\beta_{0}+\beta_{1}(\text { vacc }) \ldots+\beta_{Z} Z
$$

where variables are defined as above except for models for immunodeficiency and HIV subgroups in which

$Z=\quad$ = vector of adjustment variables including age (continuous), enrollment site, race, days from illness onset to specimen collection, date of illness onset (categorized as pre-peak, peak, or post-peak influenza periods [18]), self-reported health status (poor/fair and good/very good/excellent) and self-reported number of hospitalizations

Because we did not specifically calculate sample sizes for this study, we did a post hoc power analysis based on the observed number of cases $(n=900)$ and controls $(2600)$, vaccination rate among controls (67\%), power of $80 \%$, and a significance level of 0.05 . We determined a minimum detectable vaccine effectiveness of $20 \%$ in our overall study population during the 2017-2018 influenza season based on these assumptions.

Analyses were conducted with SAS version 9.4 software. Statistical significance was defined as a p-value $<0.05$ or a $95 \%$ confidence interval $(\mathrm{Cl})$ excluding the null value. We interpreted differences in VE estimates by IC vs non-IC subgroups, considering p-value $<0.15$ as 
statistically significant which is in line with guidance for interpreting interaction between two dichotomous variables when effect size is expected to be moderate to high[21, 22]. The study protocol was approved by the research ethics boards at the participating institutions.

\section{Results}

A total of 4,108 hospitalized adults were enrolled in HAIVEN in the 2017-2018 influenza season.

Of these, 584 were excluded because of enrollment earlier or later than the period of influenza circulation in the community $(n=259)$, missing vaccination status $(n=201)$, missing number of self-reported past year hospitalizations $(n=59)$, and other reasons $(n=65)$ (Figure 1). In the resulting dataset $(n=3,524), 1,210(34.3 \%)$ adults were identified as having an immunocompromising condition: organ transplant $(n=144,11.9 \%)$; stem cell transplant $(n=28$, $2.3 \%)$; underlying immunodeficiency $(n=49,4.0 \%)$; connective tissue and rheumatologic disease $(n=130,10.7 \%)$; chemotherapy and radiation therapy $(n=242,20 \%)$; hematologic condition ( $n=175,14.5 \%)$; chronic steroid use $(n=397,32.8 \%)$ and HIV $(n=45,3.7 \%)$.

Overall, participants were more likely to be female (56.9\%) and white (62.2\%). Mean age was 61 (SD 17.1) years, $66.7 \%$ were vaccinated, $25.8 \%$ had influenza, and $84.2 \%$ had $\geq 3$ high-risk conditions (Table 2). The IC and non-IC groups differed for several characteristics. IC participants were significantly more likely than non-IC to be of white race $(67.9 \%$ vs $59.3 \%$, $p<0.001)$, have a lower BMI (30.1 vs 31.2, $p=0.003)$, be vaccinated $(60.2 \%$ vs $54.6 \%, p=0.002)$, have a longer length of stay ( 4 vs 3 days, $p<0.001)$, have $\geq 3$ high-risk conditions $(94.2 \%$ vs $79 \%, p<0.001)$, have had $\geq 4$ hospitalizations in the previous year $(25.5 \%$ vs $19.1 \%, p<0.001)$, and present earlier in the pre-peak period $(42.1 \%$ vs $37.4 \%, p=0.02)$. IC participants were significantly less likely than non-IC participants to test positive for influenza ( $22 \%$ vs $27.8 \%$, $p<0.001)$ and to self-report their health as fair or poor (45\% vs $53.6 \%, p<0.001)$ (Table 2). 
There were 266 influenza cases in the IC adults and 644 influenza cases in non-IC adults. Most influenza infections were caused by influenza $A$, and 530 (78.8\%) were $A(H 3 N 2)$ viruses. Of 238 influenza B infections, 200 (84\%) were due to B Yamagata lineage viruses (Figure 2).

The patients in the 8 immunocompromised groups differed in sex, enrollment site, age/age group, race, BMI, influenza status, documented influenza vaccination, number of high-risk conditions, and self-reported health status, but not in the number of hospitalizations in the previous year, interval from illness onset to specimen collection, and date of illness onset (Supplementary Table S3).

Overall, vaccination was $33 \%(95 \% \mathrm{Cl}, 21 \%$ to $44 \%)$ effective in preventing hospitalization. Among IC adults, VE was $5 \%$ and not significant ( $95 \% \mathrm{Cl},-29 \%$ to $31 \%)$. VE in non-IC adults was $41 \%(95 \% \mathrm{Cl}, 27 \%$ to $52 \%)(p<0.05$ for interaction term) (Table 3$)$. VE for the different immunocompromised conditions varied widely, from $-73 \%$ for individuals with underlying immunodeficiency to $84 \%$ for stem cell transplant; however, this study was not powered to look at these subgroups and the confidence intervals varied widely (Supplementary figure S2).

\section{Discussion}

During the high-severity 2017-2018 US influenza season, we found that influenza vaccination reduced the risk of influenza-associated hospitalization among adults by $33 \%$. Overall, VE during the 2017-2018 season was lower than that estimated in previous seasons in this network $(42-54 \%)[18,23]$. The fact that influenza $A(H 3 N 2)$ viruses circulating in 2017-2018 were antigenically different from the vaccine H3N2 strain because of suspected egg-adapted glycosylation in the antigenic epitopes of the vaccines may be responsible for the lower VE [24]. As compared with VE in non-IC adults (41\%), VE in IC adults was significantly lower (5\%) during this season. This lower VE among IC adults is unlikely to be an artifact, because the findings are consistent with the immunogenicity studies of inactivated influenza vaccines (IIVs) 
that have demonstrated significantly reduced humoral immune responses to standard IIVs in immunosuppressed patients with HIV, organ transplants, cancer, and those receiving immunosuppressants [14]. In this network, influenza vaccination rate among controls was greater in the IC (60\%) than in the non-IC group (54\%), which is consistent with national US data in the insured population [25]. The higher vaccination rate among IC may be due to more frequent healthcare encounters and closer monitoring among IC patients offering more opportunities to vaccinate, or a heightened perception of risk for influenza complications by providers, leading to increased willingness to recommend influenza vaccine, and by patients, leading to greater willingness to receive vaccination.

Limited data exist on the prevention of influenza infection on immunocompromised adults by vaccination. Most studies have focused on the measurement of humoral antibody response among patients with particular immunocompromising conditions and have reported significantly reduced humoral immune responses [15, 26, 27]. However, this approach disregards the relationship between clinical outcomes and immune response, the levels of antibody titers from previous immunizations that may cause overestimations of response, nor does it consider the role of cell-mediated immune response to vaccination in the prevention of influenza infection. While studies of high dose influenza vaccine have demonstrated improved antibody responses in adult organ transplant recipients and improved antibody responses and outcomes in adults older than 65 years of age as compared to standard dose vaccine, it is unknown if enhanced vaccine options, such as high dose and adjuvanted vaccines, could improve VE in immunocompromised groups [27-30]. Increasing the evidence base for informing the use of enhanced influenza vaccines in immunosuppressed populations is necessary for determining if these interventions might offer added value to standard influenza vaccines and potentially contribute to improving efficacy of these vaccines. 
A primary challenge in the study of influenza VE in IC individuals is the definition of immunocompromise. Immunocompromising conditions are heterogenous and the degree of immunosuppression among groups is challenging to quantify. Additionally, within a defined IC group, differences in the degree of immunosuppression are difficult to assess, based on clinical records. We considered $\sim 34 \%$ of the adults hospitalized with an acute respiratory illness during the influenza season as being immunocompromised by pre-defining groups of immunocompromising conditions that were identified by ICD-10-CM codes for all medical encounters in the preceding year. To complement our case definition, we also analyzed the addition of CPT codes for chemotherapy administration, chemotherapeutic drugs recorded in the EMR, and a question at the time of enrollment about the receipt of chemotherapy or radiation therapy in the preceding 12 months. Although we did not collect other immunosuppressant and biological data, we identified a similar proportion of IC adults among those hospitalized with acute respiratory illness as identified in the study by Patel et al. that utilized MarketScan data to estimate the prevalence of immunosuppressive conditions and risk for acute respiratory illnesses [25].

Findings of this study should be interpreted in the context of several limitations. Although we used an objective and systematic mechanism to identify the different IC groups, our identification of the immunocompromising groups accounts for only a rough measure of immunosuppression. We did not consider the presence of more than one immunocompromising condition, and we were unable to evaluate the effect of timing of vaccination in relation to the immunosuppression. We were unable to evaluate VE among different IC because of inadequate sample sizes. A study with a greater number of IC adults that allows for analyses of subgroups, virus subtypes, and different vaccine formulations is needed for definitive conclusions. Our study is also limited to a single season when vaccine was mismatched to the 
circulating $\mathrm{A} / \mathrm{H} 3 \mathrm{~N} 2$ viruses and thus may not be applicable to other influenza viruses. Data are also from 4 U.S. sites and may not be generalizable.

Our study's strengths include the use of a standardized protocol with symptom-based eligibility and comprehensive PCR testing to identify influenza cases and controls, a test-negative case control design and, recruitment in geographically diverse areas. Furthermore, our study shows that immunocompromising conditions can be identified based on EMR data, without the need for cumbersome medication reviews.

Proper identification of IC groups in future VE studies will have implications for public policy development, such as a recommendation for a different vaccine formulation for IC groups, or a consideration for chemoprophylaxis for those with immunocompromise.

Vaccine effectiveness against influenza was not significant among hospitalized immunocompromised patients. In light of our findings, decreasing the burden of influenza in IC individuals may be less dependent on improving their vaccine coverage than on improving vaccination rates of close contacts of the immunocompromised individual, thereby creating a circle of protection around an IC individual. Mathematical modeling has shown that even small improvements in VE and vaccine coverage are associated with substantial reductions in influenza burden [31]. 


\section{Notes:}

HAIVEN Study Investigators: University of Pittsburgh Medical Center, Pennsylvania: Richard Zimmerman, Donald Middleton, Fernanda Silveira, Kailey Hughes, Heather Eng, Theresa Sax, Sean Saul, Charles Rinaldo, Balasubramani Goundappa, Mary Patricia Nowalk, Lori Steiffel, John Williams, Monika Johnson. Baylor Scott and White Health, Texas: Manjusha Gaglani, Kempapura Murthy, Tresa McNeal, Shekar Ghamande, Victor Escobedo, Anne Robertson, Lydia Clipper, Arundhati Rao, Kevin Chang, Marcus Volz, Kimberly Walker, Alejandro Arroliga. University of Michigan, Michigan: Arnold Monto, Emily Martin, Ryan Malosh, Joshua Petrie, Adam Lauring, Caroline Cheng, Hannah Segaloff, E. J. McSpadden, Emileigh Johnson, Rachel Truscon. Henry Ford Health System, Michigan: Lois Lamerato, Susan Davis, Marcus Zervos. Vanderbilt University Medical Center, Tennessee: H. Keipp Talbot, Dayna Wyatt, Yuwei Zhu, Zhouwen Liu, Rendie McHenry, Natasha Halasa, Sandra Alvarez Calvillo, Stephanie Longmire, Laura Stewart. CDC: Jill Ferdinands, Alicia Fry, Elif Alyanak, Emily Smith, Courtney Strickland, Sarah Spencer, Brendan Flannery, Jessie Chung, Xiyan Xu, Stephen Lindstrom, LaShondra Berman, Wendy Sessions, Rebecca Kondor, Manish Patel.

Disclaimer: The findings and conclusions in this report are those of the authors and do not necessarily represent the official position of CDC.

Financial Support: This study was funded by the CDC (cooperative agreement IP15-002). Vanderbilt also received support from CTSA award $n^{\circ}$. UL1 TR002243 from the National Center for Advancing Translational Sciences.

\section{Potential Conflicts of Interest:}

DBM has received personal fees from Sequris, Pfizer, and Sanofi Pasteur, and grants from Pfizer. JF reports non-financial support from the Institute for Influenza Epidemiology. RKZ has 
received grants from Sanofi Pasteur and Merck \& Co. All other authors report no potential conflicts.

\section{References}

1. Harpaz R, Dahl RM, Dooling KL. Prevalence of Immunosuppression Among US Adults, 2013. JAMA 2016; 316(23): 2547-8.

2. Rubin LG, Levin MJ, Ljungman P, et al. 2013 IDSA clinical practice guideline for vaccination of the immunocompromised host. Clin Infect Dis 2014; 58(3): e44-100.

3. Dropulic LK, Lederman HM. Overview of Infections in the Immunocompromised Host. Microbiol Spectr 2016; 4(4).

4. Centers for Disease Control and Prevention. Past Seasons Estimated Influenza Disease Burden. Available at: https://www.cdc.gov/flu/about/burden/past-seasons.html. Accessed July 30 .

5. Kumar D, Michaels MG, Morris MI, et al. Outcomes from pandemic influenza A H1N1 infection in recipients of solid-organ transplants: a multicentre cohort study. Lancet Infect Dis $2010 ; 10(8): 521-6$.

6. Ljungman P, de la Camara R, Perez-Bercoff L, et al. Outcome of pandemic H1N1 infections in hematopoietic stem cell transplant recipients. Haematologica 2011; 96(8): 1231-5.

7. Chemaly RF, Vigil KJ, Saad M, et al. A multicenter study of pandemic influenza A (H1N1) infection in patients with solid tumors in 3 countries: early therapy improves outcomes. Cancer 2012; 118(18): 4627-33.

8. Memoli MJ, Athota R, Reed S, et al. The natural history of influenza infection in the severely immunocompromised vs nonimmunocompromised hosts. Clin Infect Dis 2014; 58(2): 214-24. 
9. Garnacho-Montero J, Leon-Moya C, Gutierrez-Pizarraya A, et al. Clinical characteristics, evolution, and treatment-related risk factors for mortality among immunosuppressed patients with influenza $A(\mathrm{H} 1 \mathrm{~N} 1)$ virus admitted to the intensive care unit. J Crit Care 2018; 48: 172-7.

10. Collins JP, Campbell AP, Openo K, et al. Outcomes of Immunocompromised Adults Hospitalized With Laboratory-confirmed Influenza in the United States, 2011-2015. Clin Infect Dis 2020; 70(10): 2121-30.

11. Grohskopf LA, Alyanak E, Broder KR, Walter EB, Fry AM, Jernigan DB. Prevention and Control of Seasonal Influenza with Vaccines: Recommendations of the Advisory Committee on Immunization Practices - United States, 2019-20 Influenza Season. MMWR Recomm Rep 2019; 68(3): 1-21.

12. Blanchette PS, Chung $\mathrm{H}$, Pritchard KI, et al. Influenza Vaccine Effectiveness Among Patients With Cancer: A Population-Based Study Using Health Administrative and Laboratory Testing Data From Ontario, Canada. J Clin Oncol 2019; 37(30): 2795-804.

13. Nichols MK, Andrew MK, Hatchette TF, et al. Influenza vaccine effectiveness to prevent influenza-related hospitalizations and serious outcomes in Canadian adults over the 2011/12 through 2013/14 influenza seasons: A pooled analysis from the Canadian Immunization Research Network (CIRN) Serious Outcomes Surveillance (SOS Network). Vaccine 2018; 36(16): 2166-75.

14. Beck CR, McKenzie BC, Hashim AB, et al. Influenza vaccination for immunocompromised patients: systematic review and meta-analysis by etiology. J Infect Dis 2012; 206(8): 1250-9.

15. Watcharananan SP, Thakkinstian A, Srichunrasmee C, Chuntratita W, Sumethkul V. Comparison of the immunogenicity of a monovalent influenza $A / H 1 N 12009$ vaccine between healthy individuals, patients with chronic renal failure, and immunocompromised populations. Transplant Proc 2014; 46(2): 328-31. 
16. Coudeville L, Bailleux F, Riche B, Megas F, Andre P, Ecochard R. Relationship between haemagglutination-inhibiting antibody titres and clinical protection against influenza: development and application of a bayesian random-effects model. BMC Med Res Methodol 2010; 10: 18.

17. Hannoun C, Megas F, Piercy J. Immunogenicity and protective efficacy of influenza vaccination. Virus Res 2004; 103(1-2): 133-8.

18. Ferdinands JM, Gaglani M, Martin ET, et al. Prevention of Influenza Hospitalization Among Adults in the United States, 2015-2016: Results From the US Hospitalized Adult Influenza Vaccine Effectiveness Network (HAIVEN). J Infect Dis 2019; 220(8): 1265-75.

19. Greenberg JA, Hohmann SF, Hall JB, Kress JP, David MZ. Validation of a Method to Identify Immunocompromised Patients with Severe Sepsis in Administrative Databases. Ann Am Thorac Soc 2016; 13(2): 253-8.

20. Foppa IM, Haber M, Ferdinands JM, Shay DK. The case test-negative design for studies of the effectiveness of influenza vaccine. Vaccine 2013; 31(30): 3104-9.

21. Durand CP. Does raising type 1 error rate improve power to detect interactions in linear regression models? A simulation study. PLoS One 2013; 8(8): e71079.

22. Marshall SW. Power for tests of interaction: effect of raising the Type I error rate. Epidemiol Perspect Innov 2007; 4: 4.

23. Tenforde MW, Chung J, Smith ER, et al. Influenza vaccine effectiveness in inpatient and outpatient settings in the United States, 2015 - 2018. Clin Infect Dis 2020.

24. Levine MZ, Martin ET, Petrie JG, et al. Antibodies Against Egg- and Cell-Grown Influenza A(H3N2) Viruses in Adults Hospitalized During the 2017-2018 Influenza Season. J Infect Dis 2019; 219(12): 1904-12.

25. Patel M, Chen J, Kim S, et al. Analysis of MarketScan Data for Immunosuppressive Conditions and Hospitalizations for Acute Respiratory IIIness, United States. Emerg Infect Dis 2020; 26(8): 1720-30. 
26. Helantera I, Janes R, Anttila VJ. Clinical efficacy of seasonal influenza vaccination: characteristics of two outbreaks of influenza $A(\mathrm{H} 1 \mathrm{~N} 1)$ in immunocompromised patients. J Hosp Infect 2018; 99(2): 169-74.

27. Natori Y, Shiotsuka M, Slomovic J, et al. A Double-Blind, Randomized Trial of High-Dose vs Standard-Dose Influenza Vaccine in Adult Solid-Organ Transplant Recipients. Clin Infect Dis 2018; 66(11): 1698-704.

28. DiazGranados CA, Dunning AJ, Kimmel M, et al. Efficacy of high-dose versus standarddose influenza vaccine in older adults. N Engl J Med 2014; 371(7): 635-45.

29. Doyle JD, Beacham L, Martin ET, et al. Relative and absolute effectiveness of high-dose and standard-dose influenza vaccine against influenza-related hospitalization among older adults - United States, 2015-2017. Clin Infect Dis 2020.

30. Young-Xu Y, Van Aalst R, Mahmud SM, et al. Relative Vaccine Effectiveness of HighDose Versus Standard-Dose Influenza Vaccines Among Veterans Health Administration Patients. J Infect Dis 2018; 217(11): 1718-27.

31. Hughes MM, Reed C, Flannery B, et al. Projected Population Benefit of Increased Effectiveness and Coverage of Influenza Vaccination on Influenza Burden in the United States. Clin Infect Dis 2020; 70(12): 2496-502. 


\section{Tables}

Table 1: ICD-10 and CPT codes used to classify the immunocompromised group

\begin{tabular}{|l|l|}
\hline $\begin{array}{l}\text { Immunocompromised } \\
\text { Group }\end{array}$ & ICD-10 Codes \\
\hline Organ Transplant & $\begin{array}{l}\text { T86.1, T86.2, T86.3, T86.4, T86.81, T86.85, Z48.2, Z94.0, } \\
\text { Z94.1, Z94.2, Z94.3, Z94.4, Z94.82, Z94.83 }\end{array}$ \\
\hline Stem Cell Transplant & Z94.84, T86.0, T86.5 \\
\hline $\begin{array}{l}\text { Underlying } \\
\text { Immunodeficiency }\end{array}$ & $\begin{array}{l}\text { D80, D81.0, D81.1, D81.9, D82, D83, D84, D84.1, D84.8, } \\
\text { D84.9, D89.8, D89.9 }\end{array}$ \\
\hline $\begin{array}{l}\text { Connective Tissue } \\
\text { Disorder }\end{array}$ & $\begin{array}{l}\text { M05, M06, M 08.0, M 08.2, M08.3, M08.4, L40.54, L40.59, M32, } \\
\text { M30.0, M30.1, M30.2, M31.3, M33, M34, M34.0, M34.1, M34.9, } \\
\text { M35.0, M35.9 }\end{array}$ \\
\hline $\begin{array}{l}\text { Chemotherapy/ Radiation } \\
\text { Therapy }\end{array}$ & Z51.0, Z51.1 \\
\hline Hematologic Conditions & C95.00, C95.10, D61.0, D61.2, D61.9, D70, D71, D72, D73.0, \\
& C81, C82, C83, C84, C85, C86, C88, C90, C91, C92, C93, C94, \\
\hline C96, D46
\end{tabular}

CPT codes for chemotherapy and radiation therapy were collected at the Pennsylvania sites only. 
Table 2: Patient characteristics overall by immunocompromising condition, US Hospitalized

Adult Influenza Vaccine Effectiveness (HAIVEN) study, 2017-2018 ( $\mathrm{n}=3,524)$

\begin{tabular}{|c|c|c|c|c|}
\hline & $\begin{array}{c}\text { Total } \\
(n=3,524)\end{array}$ & $\begin{array}{c}\text { Non- } \\
\text { Immunocompromised } \\
(n=2,314)\end{array}$ & $\begin{array}{l}\text { Immunocompromised } \\
\qquad(\mathrm{n}=1,210)\end{array}$ & $\begin{array}{c}\mathrm{p}- \\
\text { value }\end{array}$ \\
\hline \multicolumn{5}{|l|}{ Enrollment site, $\mathrm{n}(\%)$} \\
\hline Michigan & $943(26.8)$ & 714 (30.9) & $229(18.9)$ & $<0.001$ \\
\hline Pennsylvania & $834(23.7)$ & $571(24.7)$ & $263(21.7)$ & \\
\hline Tennessee & $589(16.7)$ & $369(16.0)$ & $220(18.2)$ & \\
\hline Texas & $1158(32.9)$ & $660(28.5)$ & $498(41.2)$ & \\
\hline Female, n (\%) & $2004(56.9)$ & $1317(56.9)$ & $687(56.8)$ & 0.94 \\
\hline \multicolumn{5}{|l|}{ Age group, $\mathrm{n}(\%)$} \\
\hline $18-49$ & $790(22.4)$ & $534(23.1)$ & $256(21.2)$ & 0.37 \\
\hline $50-64$ & $1173(33.3)$ & $753(32.5)$ & $420(34.7)$ & \\
\hline $65-74$ & $798(22.6)$ & $517(22.3)$ & $281(23.2)$ & \\
\hline $75+$ & $763(21.7)$ & $510(22.0)$ & $253(20.9)$ & \\
\hline Age, mean \pm SD & $61.0 \pm 17.1$ & $60.8 \pm 17.7$ & $61.4 \pm 15.9$ & 0.29 \\
\hline \multicolumn{5}{|l|}{ Race, n (\%) } \\
\hline White, non-Hispanic & $2193(62.2)$ & $1371(59.3)$ & $822(67.9)$ & $<0.001$ \\
\hline Non-white & $1331(37.8)$ & $943(40.8)$ & $388(32.1)$ & \\
\hline $\mathrm{BMI}$, mean $\pm \mathrm{SD}$ & $30.8 \pm 9.5$ & $31.2 \pm 9.8$ & $30.1 \pm 8.9$ & 0.001 \\
\hline \multicolumn{5}{|l|}{ Any flu, n (\%) } \\
\hline Negative & $2614(74.2)$ & $1670(72.2)$ & $944(78.0)$ & $<0.001$ \\
\hline Positive & $910(25.8)$ & $644(27.8)$ & $266(22.0)$ & \\
\hline \multicolumn{5}{|c|}{ Documented vaccination, $\mathrm{n}(\%)$} \\
\hline No & 1174 (33.3) & $805(34.8)$ & $369(30.5)$ & 0.01 \\
\hline
\end{tabular}




\begin{tabular}{|c|c|c|c|c|}
\hline Yes & $2350(66.7)$ & 1509 (65.2) & $841(69.5)$ & \\
\hline Length of stay, median (IQR) & $3.0(4.0)$ & $3.0(3.0)$ & $4.0(4.0)$ & $<0.001$ \\
\hline \multicolumn{5}{|l|}{ Influenza-like illness (ILI) } \\
\hline \multicolumn{5}{|l|}{ symptoms, n (\%) } \\
\hline No & $1208(34.3)$ & $797(34.4)$ & $411(34.0)$ & 0.78 \\
\hline Yes & $2316(65.7)$ & $1517(65.6)$ & $799(66.0)$ & \\
\hline \multirow{2}{*}{\multicolumn{5}{|c|}{$\begin{array}{l}\text { Number of high-risk conditions, } \\
\mathrm{n}(\%)\end{array}$}} \\
\hline & & & & \\
\hline No high-risk conditions & $162(4.6)$ & $141(6.1)$ & $21(1.7)$ & $<0.001$ \\
\hline 1-2 high-risk conditions & $394(11.2)$ & $345(14.9)$ & $49(4.1)$ & \\
\hline$\geq 3$ high-risk conditions & $2968(84.2)$ & $1828(79.0)$ & $1140(94.2)$ & \\
\hline \multirow{2}{*}{\multicolumn{5}{|c|}{$\begin{array}{l}\text { Self-reported hospitalizations in } \\
\text { the prior year, n (\%) }\end{array}$}} \\
\hline & & & & \\
\hline 0-3 hospitalizations & $2773(78.7)$ & $1871(80.9)$ & $902(74.6)$ & $<0.001$ \\
\hline$\geq 4$ hospitalizations & $751(21.3)$ & $443(19.1)$ & $308(25.5)$ & \\
\hline \multicolumn{5}{|l|}{ Interval from illness onset and } \\
\hline \multicolumn{5}{|l|}{ specimen collection, n (\%) } \\
\hline $0-1$ days & $693(19.7)$ & $435(18.8)$ & $258(21.3)$ & 0.14 \\
\hline 2-4 days & $1621(46.0)$ & $1065(46.0)$ & $556(46.0)$ & \\
\hline $5-10$ days & $1210(34.3)$ & $814(35.2)$ & $396(32.7)$ & \\
\hline \multicolumn{5}{|l|}{ Onset date } \\
\hline Pre-peak & $1374(39.0)$ & $865(37.4)$ & $509(42.1)$ & 0.02 \\
\hline Peak & $844(24.0)$ & $559(24.2)$ & $285(23.6)$ & \\
\hline Post-peak & $1306(37.1)$ & $890(38.5)$ & $416(34.4)$ & \\
\hline \multicolumn{5}{|l|}{ Self-reported health status, $n$} \\
\hline$(\%)$ & & & & \\
\hline
\end{tabular}


medRxiv preprint doi: https://doi.org/10.1101/2020.10.08.20208579; this version posted October 13, 2020. The copyright holder for this preprint (which was not certified by peer review) is the author/funder, who has granted medRxiv a license to display the preprint in perpetuity. It is made available under a CC-BY-NC-ND 4.0 International license.

\begin{tabular}{|lllll|} 
Excellent/ very good/good & $1739(49.4)$ & $1074(46.4)$ & $665(55.0)$ & $<0.001$ \\
Fair/ poor & $1785(50.7)$ & $1240(53.6)$ & $545(45.0)$ & \\
\hline
\end{tabular}


Table 3: Influenza vaccine effectiveness for prevention of influenza A or B-associated hospitalizations in immunocompromised and non-immunocompromised adults, US Hospitalized Adult Influenza Vaccine Effectiveness (HAIVEN) study, 2017-2018

\begin{tabular}{|c|c|c|c|}
\hline & $\begin{array}{c}\mathrm{N} \text { Influenza } \\
\text { Cases (\% } \\
\text { vaccinated) }\end{array}$ & $\begin{array}{c}\text { Unadjusted VE, } \\
\%(95 \% \mathrm{Cl})\end{array}$ & $\begin{array}{l}\text { Adjusted VE, \% } \\
\qquad(95 \% \mathrm{Cl})^{*}\end{array}$ \\
\hline All, $n=3524$ & $910(67)$ & $28(16,38)$ & $33(21,44)$ \\
\hline $\begin{array}{l}\text { Non-immunocompromised, } \\
n=2314\end{array}$ & $644(65)$ & $36(23,47)$ & $41(27,52)$ \\
\hline $\begin{array}{l}\text { Immunocompromised, } \\
\mathrm{n}=1210\end{array}$ & $266(70)$ & $-0.3(-35,25)$ & $5(-29,31)$ \\
\hline $\begin{array}{l}\text { Non-immunocompromised, } \\
\text { influenza A }\end{array}$ & $471(65)$ & $31(15,44)$ & $31(14,46)$ \\
\hline $\begin{array}{l}\text { Immunocompromised, } \\
\text { influenza A }\end{array}$ & $202(70)$ & $1(-37,29)$ & $4(-36,32)$ \\
\hline $\begin{array}{l}\text { Non-immunocompromised, } \\
\text { H1N1 }\end{array}$ & $71(65)$ & $57(32,74)$ & $52(19,71)$ \\
\hline Immunocompromised, H1N1 & $33(70)$ & $60(17,80)$ & $51(-2,77)$ \\
\hline $\begin{array}{l}\text { Non-immunocompromised, } \\
\text { H3N2 }\end{array}$ & $369(65)$ & $21(0,37)$ & $23(0,40)$ \\
\hline Immunocompromised, H3N2 & $161(70)$ & $-28(-87,12)$ & $-18(-75,20)$ \\
\hline $\begin{array}{l}\text { Non-immunocompromised, } \\
\text { influenza B }\end{array}$ & $173(65)$ & $34(10,52)$ & $45(22,61)$ \\
\hline $\begin{array}{l}\text { Immunocompromised, } \\
\text { influenza B }\end{array}$ & $65(70)$ & $1(-70,43)$ & $13(-52,51)$ \\
\hline
\end{tabular}

${ }^{*}$ Adjusted for enrolling site, onset date (pre-peak, peak, post-peak), age, race, days from illness onset to specimen collection (0-1, 2-4, 5-10 days), self-reported health (poor/fair, good/very good/excellent), and self-reported hospitalizations. 
medRxiv preprint doi: https://doi.org/10.1101/2020.10.08.20208579; this version posted October 13, 2020. The copyright holder for this preprint (which was not certified by peer review) is the author/funder, who has granted medRxiv a license to display the preprint in perpetuity.

\section{It is made available under a CC-BY-NC-ND 4.0 International license.}

Figure 1: US Hospitalized Adult Influenza Vaccine Effectiveness Network (HAIVEN) study population, 2017-2018.

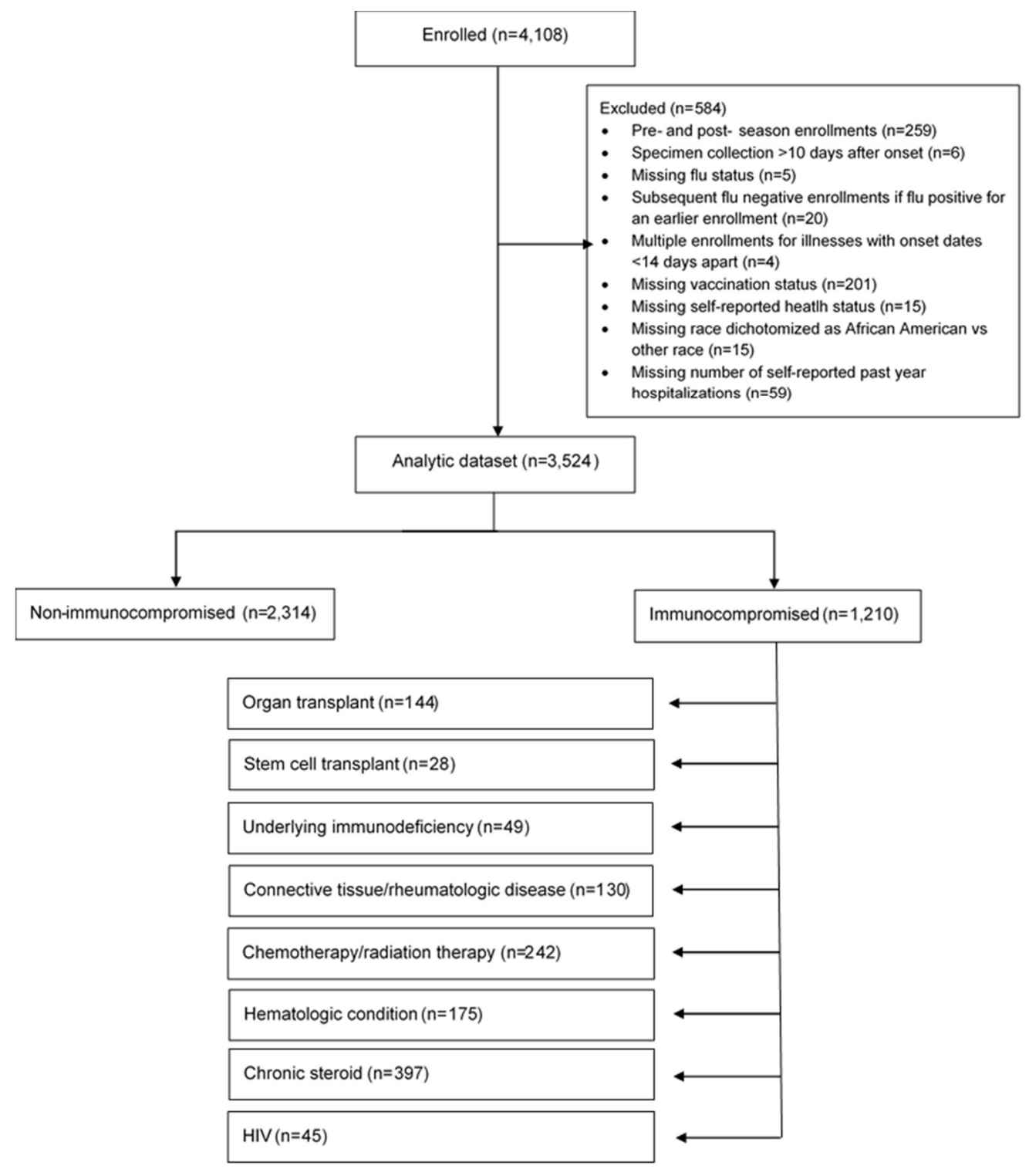

Figure 1 legend: Immunocompromised groups were mutually exclusive and followed the order listed here. As an example, if an individual had an ICD-10 code that classified him/her as an organ transplant, this individual was grouped in this category, even if he/she also had an ICD-10 for chemotherapy. 
medRxiv preprint doi: https://doi.org/10.1101/2020.10.08.20208579; this version posted October 13, 2020. The copyright holder for this preprint (which was not certified by peer review) is the author/funder, who has granted medRxiv a license to display the preprint in perpetuity.

\section{It is made available under a CC-BY-NC-ND 4.0 International license .}

Figure 2: Influenza virus type/subtype and lineage in the non-immunocompromised and

\section{immunocompromised groups}

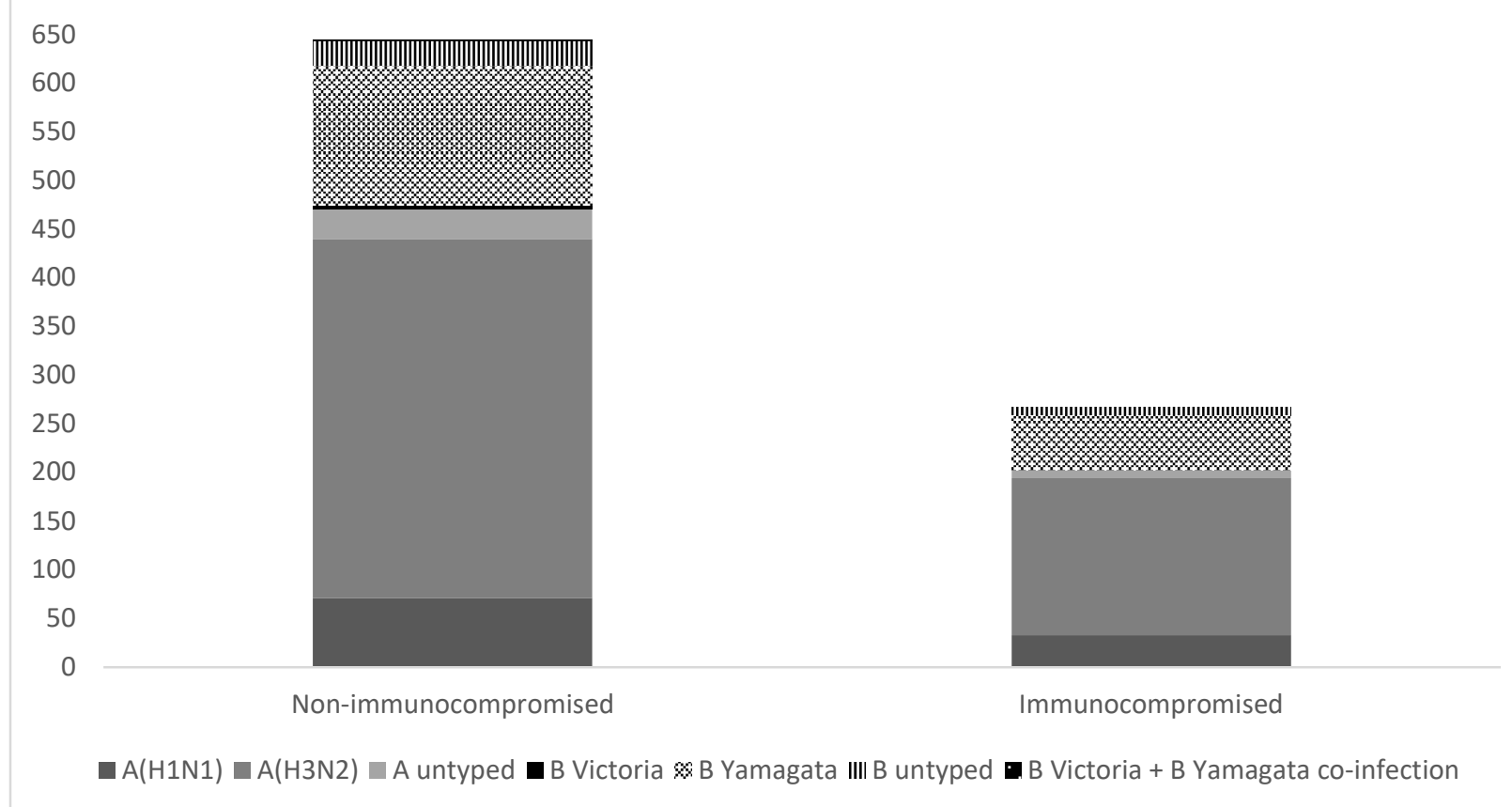

